\title{
Direct medication delivery modifies the periodontal biofilm
}

\author{
Duane C. Keller ${ }^{*}$ and Marissa Buechel
}

*Correspondence: drdkeller@sbcglobal.net

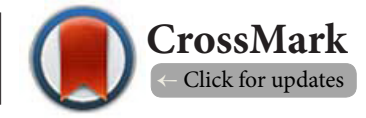

Keller Professional Group PC, Perio Protect LLC, Bayless Avenue, St. Louis, MO, USA.

\begin{abstract}
Background: Conventional periodontal treatment is inadequate in controlling the periodontal biofilm. This is due to the nature and size of the bacteria, biofilm adaptive and reproductive capacity and an inability to mechanically remove the bacteria. The composition at the beginning of conventional treatment is the same as at the end.

Method: Management involving a direct medication delivery of hydrogen peroxide, oxygen and an antioxidant (Perio Tray, Perio Protect LLC St. Louis, MO) was evaluated by scanning electron microscopy imagery (SEM) and DNA analysis to determine what bacteria were present before, during and after treatment. The biofilm by SEM analysis was changed in the number of bacteria while the DNA analysis demonstrated changes in the biofilm constituency.

Results: The biofilm composition shifted from predominantly Gram negative obligate anaerobes before treatment to Gram positive anaerobes, Gram positive and negative facultative anaerobes and aerobic bacteria during the first month to the end of treatment. There was a $-\log ^{2}$ to a $-\log ^{4}$ change in the number of bacteria during the course of the treatment.

Conclusion: This study indicates that direct medication delivery of medicaments with a custom formed tray modifies the biofilm from a more virulent anaerobic to a less virulent aerobic composition and reduces the number of pathogens. Fewer bacteria that are less virulent should provide better treatment results.
\end{abstract}

Keywords: Biofilm, periodontal disease, direct medication delivery, DNA analysis, SEM analysis, perio tray, perio gel

\section{Introduction}

Most bacteria are found to live in a biofilm as compared to living as planktonic bacteria [1]. A biofilm is a mixture of many species of bacteria along with fungi, viruses, etc. Over 500 species have been identified in typical dental plaque biofilm formation and they have a similarity of genetic material that enables cell to cell communication [2]. Biofilms form a protective matrix which is held together by sugary lipid materials, collectively termed "extracellular polymeric substances" or "EPS" which allows the biofilms to form complex three-dimensional, resilient, attached communities [3].

The biofilm matrix physically protects the biofilm from physical injury and is difficult for many materials to penetrate. Antibiotics are less effective as most antibiotics have a difficult time penetrating the matrix. When one bacteria develops a resistance to an antibiotic, this can be "shared" with other bacteria through cell-to-cell communications like horizontal gene transfer, etc., which fosters other bacteria developing antibiotic resistance and an increased ability to adapt [4].

The distribution of the different bacterial species within the oral biofilm is not random. Most species prefer certain sites over others. Aerobic bacteria prefer the region where there is increased oxygen. Facultative bacteria are often found in the transition zone between oxygen presence and oxygen depletion. Facultative anaerobes can use up the oxygen providing an environment where obligate anaerobes may become the predominant species such as deeper periodontal pocket [5]. The various bacteria communicate, correlate and affect their environment. The micro-environmental differences affect the bacteria [6], and a difference in the bacteria virulence may affect the host. Therefore, knowing which bacteria are present in the biofilm may facilitate treatment and an improved host response.

Schaudinn et al., demonstrate the use of polycarboxylate carriers and paper point analyses in evaluating the biofilm in patients 
Keller et al. Oral Biology and Dentistry 2017,

http://www.hoajonline.com/journals/pdf/2053-5775-5-1.pdf

doi: 10.7243/2053-5775-5-1

periodontal pockets. They demonstrate the placement of a sterile carrier that is adhered supra-gingival to the crown of the tooth so that the carrier remains in the patients infected periodontal pocket. This allows the subgingival biofilm to grow on the sterilized carrier so that the carrier and the attached biofilm can be removed at specific times and analyzed by scanning electron microscopy. The carrier did not modify the biofilm; rather the biofilm that grew on the carrier was the same as the biofilm from paper points acquired from the same location [25].

Various periodontal bacteria have been evaluated, to determine their role in the progression of disease [7]. Multiple factors are involved in bacterial virulence such as surface structure adaptations, metabolic function, interaction with host defenses and the ability to cause tissue damage and inflammation. Pathogenic bacteria associated with chronic infections have been found to more closely resemble the proteome of humans, whereas bacteria causing acute infections are less similar to the human proteome [8]. Gram negative obligate anaerobes increase virulence through adhesins for host cell attachment, production of metabolic interdependent associations, the production of capsules and surface characteristics that inhibit phagocytosis, the production of lipopolysaccharides, proteases and complement components and hydrolyzing enzymes [9].

Gram negative obligate anaerobes are more virulent bacteria associated with periodontal disease and it has been shown that for the most part, Gram positive anaerobes are components of the healthy flora of the oral mucosal surfaces and have a relatively low virulence involvement [10]. Gram positive anaerobes are often mixed with other facultative anaerobes and aerobic bacteria and are most often involved with soft tissue infections of the skin, chronic otitis media and sinusitis, aspiration pneumonia, intra-abdominal infections and some dental infections [11]. Gram negative obligate anaerobes are the predominant species in most dental and systemic infections and outnumber the facultative bacteria and aerobic bacteria with ratios ranging from 10:1 to 10,000:1 [12].

Conventional mechanical treatment and conventional homecare do not change the species of bacteria present before and after treatment [13]. Colombo et al., found mechanical therapy modestly decreased the mean counts of many of the recognized periodontal pathogens, but the counts of other suspected pathogens increased after treatment and the mean frequency of the reduced pathogens increased to base values by 9 months [14]. Palmer et al., found that removal of $50 \%$ of a biofilm mass resulted in a regrowth that reached $400 \%$ regeneration within three hours. [15] Teles et al., found that biofilms following mechanical therapy attained a growth potential that superseded pre-treatment levels within days [16]. Teles et al., demonstrated there was no significant alteration in the proportions of periodontal pathogens following conventional plaque removal [17].

Effective periodontal treatment involving decreased tis- sue damage would reduce the numbers of virulent bacteria that would be replaced with fewer, less virulent bacteria. It is equally important that the patient is able to maintain plaque control. Conventional homecare (tooth brush) can reduce the number of supragingival bacteria [18], but the use of a tooth brush does not significantly alter the prevalence of subgingival bacteria [19]. Mechanical treatment and the effect of conventional homecare fail to adequately reduce plaque over time and repeated subgingival scaling and root planing should be avoided in sites that are $3 \mathrm{~mm}$ or less as this is liable to traumatize the periodontium [20]. Successful homecare must provide a means to successfully manage biofilm re-development

A variety of factors including oxygen levels affect the biofilm re-development following mechanical therapy. Cellular interactions like oxygen metabolism demonstrate both inhibitory pathways and facilitation pathways between various bacteria within a biofilm, especially as it affects obligate anaerobic species. Obligate anaerobes have difficulty living in an oxygen rich environment. Many of the aerobic species as well as facultative anaerobes consume oxygen resulting in a lower oxygen concentration. Fusobacterium nucleatum is one example which can aggregate with both aerobic and obligate anaerobes, fostering the number of obligate anaerobes [21]. When most of the oxygen is consumed in a periodontal pocket, the Gram negative obligate anaerobes increase within the biofilm [22] and this increase in Gram negative obligate anaerobes such as Porphyromonas gingivalis would cause an increase in virulence, resulting in a greater host inflammatory response [23].

The hypothesis that adding oxygen into periodontal pockets would have a positive effect on host tissue inflammatory response was initially examined by Schaudinn $C$ et al., who used and reported on the biofilm potential method to assess the ecological status of periodontal sulci with respect to the health and spreading tendencies of the biofilm community. Their data suggested the biofilm potential is an accurate indicator of determining microbiologic health of the sulcus [24] and that oxidative chemical strategies such as those used with the direct medication delivery of hydrogen peroxide and an antioxidant are effective in modifying the biofilm [25]. Their study used a Perio Tray to deliver the hydrogen peroxide, oxygen and Vibramycin into the periodontal pocket. The amount of hydrogen peroxide delivered with the Perio Tray was evaluated by Dunlap et al. This research determined 0.7 grams of hydrogen peroxide and a subclinical dose of Vibramycin were delivered subgingival and interproximal per use resulting in a $5.7 \mathrm{X}$ oxygen saturation [26].

The introduction of hydrogen peroxide, oxygen and Vibramycin reduced the number of bacteria in the biofilm. Modifying the biofilm involves measuring a shift in the number and type of bacteria using SEM images and DNA analysis. Polycarboxylate carriers were inserted into patient's periodontal pockets before treatment and at regular intervals during treatment. The samples were removed and inspected 
to ascertain the number and the appearance of the bacteria per unit area using the SEM analysis. The number of bacteria was demonstrated to decrease per unit area by a $-\log ^{2-4}$. This demonstrated a change in the number of bacteria (Figures 1-4), but it did not clarify which bacteria were affected.

Changes in the type of bacteria present in the biofilm could be evaluated through DNA analysis by comparing initial bacterial samples taken before treatment with samples taken during and after treatment. The DNA analysis identifies microbes that comprise $2 \%$ or more of the biofilm from the patient sample. The initial samples were taken six to twelve months after conventional mechanical treatment and homecare. These initial samples were compared to during and after treatment samples to ascertain changes in the biofilm constituents in response to direct medication delivery.

Changing the type of bacteria present in periodontal pockets may have an effect on virulence. It is also possible to modify virulence by affecting the concentration of oxygen in the biofilm [27]. The Perio Tray delivers hydrogen peroxide into the periodontal pocket where it increases the periodontal pocket oxygen concentration.

Studies have shown that anaerobes and aerobes can survive in the presence of oxygen by virtue of an elaborate system of defenses involving key enzymes used to control oxygen metabolism. Anaerobic and aerobic bacterial enzymes are adversely affected by hydrogen peroxide so the bacteria are unable to maintain a favorable oxygen balance in the presence of hydrogen peroxide [28].

\section{Materials and methods}

The literature is replete with research that shows conventional treatment and homecare are unable to adequately control periodontal disease. This is explained in part by the inability of scaling and root planing, surgery and conventional homecare to remove all of the biofilm [29]. The biofilm regeneration after conventional care lead, Chandki R, et al., to state: "The need of the hour is to supplement the conventional treatment strategies like Scaling, Root planing and Surgery with Chemical Plaque Control Agents [30]." Teles FR et al., show that the biofilm at the end of conventional treatment has almost the exact same constituency as the biofilm prior to treatment [31]. The remaining biofilm bacteria have only to regrow which helps explain the disease reoccurrence and why patients are routinely rescheduled at specific times for re-examination and re-treatment.

The patients in this study had all received conventional treatment and home care (debridement, scaling and root planing, prophylaxis, home care instructions, tooth paste, oral rinse, floss and mechanical tooth brush) within the prior 6 to 12 months. During the re-examination procedures (6 to 12 months after conventional care), it was discovered that the patients had a reoccurrence of the disease such as periodontal pockets, bleeding upon probing, swelling and redness. The biofilm samples taken before any treatment were comprised of the biofilm populations that remained or regrew after conventional mechanical treatments and homecare.

Patients were examined with a periodontal probing, radiographs and a clinical evaluation was performed to attain a diagnosis of periodontal disease. Following signing an informed consent form, impressions were taken for a prescription tray (Perio Tray, Perio Protect, St. Louis, MO) that is fabricated according to the patient's pre-treatment conditions.

Polycarboxylate carriers were inserted into patient's periodontal pocket before treatment and at regular intervals during treatment. The samples were removed and evaluated 'by SEM analysis to determine the number and the appearance of the bacteria per unit area. The number of bacteria prior to treatment was compared to the number of bacteria found during treatment, and the number of bacteria was found to decrease by $95 \%$ to $99+\%$ (Schaudinn et al., [25]), but it did not clarify which bacteria were affected. No polycarboxylate carrier site was used for DNA analysis.

The initial samples were taken for DNA analysis at the tray insertion visit to minimize site disturbance and maximize sample accuracy. A sterile cannula tip was attached to a sterile syringe. The tip was placed into the bottom of the patient's periodontal pocket and the plunger was elevated to create a negative pressure inside the syringe and cannula. The biofilm at the tip of the cannula was suctioned into the cannula, which was held in place for 10 seconds, then removed.

The sample collection tip was immediately placed into a sterile transfer container and sealed to improve specimen management and decrease possible errors. The sample was shipped for processing in accordance with the laboratory's prescribed recommendations. Analysis of the sample provided a list of all bacteria and fungi which represented at least $2 \%$ of the biofilm population. The percentage of bacteria/gram was computed and compared during and after treatment as: Heavy $=10^{8}$ or greater

Medium $=10^{6}$ to $10^{7}$

Low $=10^{5}$ or less

Detected bacteria were listed as a percentage of the DNA found in the sample belonging to the specific microbe. Bacteria $<2 \%$ were not reported.

Example of Detected bacteria

Porphyromonas gingivalis $64 \%$

Fusobacterium nucleatum 22\%

Tannerella forsythia $8 \%$

The Perio Tray delivers $0.70 \mathrm{gm} /$ tray of $1.7 \%$ hydrogen peroxide gel (Perio Gel) and a subclinical dose (3 drops or less / tray) of doxycycline (Vibramycin Syrup) subgingival and interproximal. The tray holds the medication in the periodontal pocket for 15 minutes. This method increases oxygen saturation 5.7 X in the periodontal pocket [32]. The Vibramycin was utilized for the antioxidant function of the medication.

The patients started using their Perio Tray 3 to 4 times a day for 15 minutes for the first two weeks in accordance with the Perio Protect Method: 


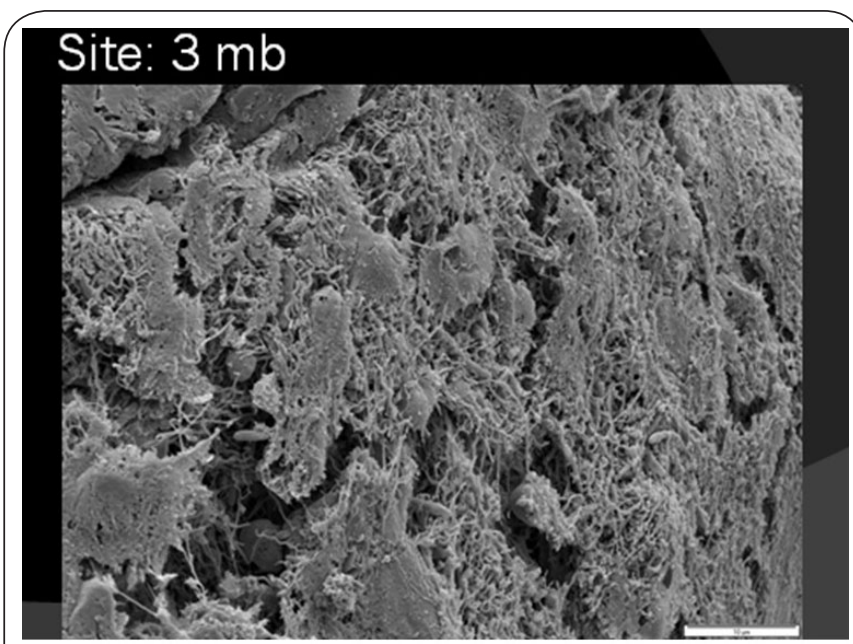

Figure 1. Is a scanning electron microscopic presentation of the bacteria found in the mesial buccal pocket of tooth \#3. These are bacteria that are present six to twelve months following conventional mechanical care and homecare. The bacteria following conventional treatment and homecare can be compared to the bacteria 1 month after direct medication treatment (Figure 2).

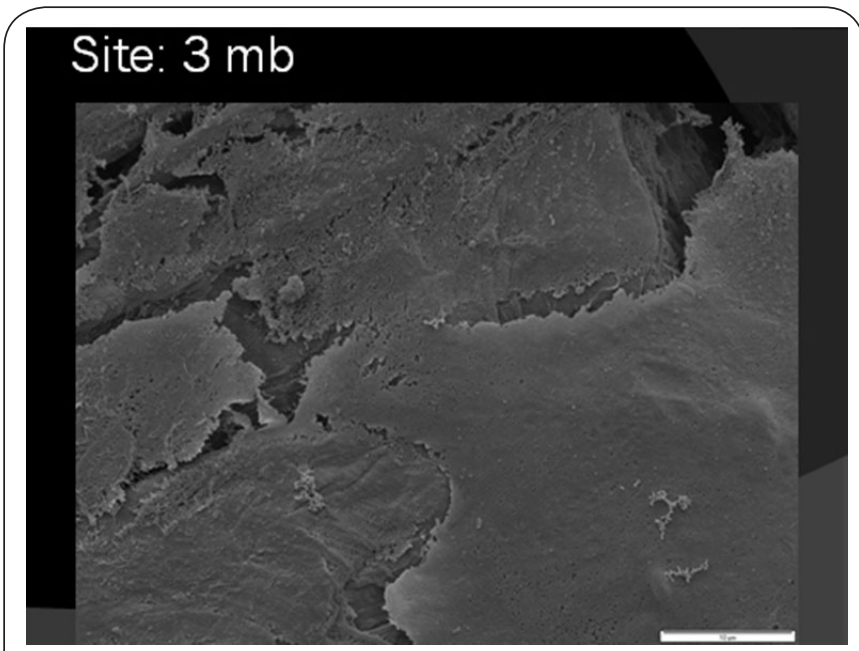

Figure 2. Is a SEM analysis of the bacteria found in the mesial buccal periodontal pocket of tooth \#3. The direct medication method delivered hydrogen peroxide gel (Perio Gel) for one month. The composite of bacteria has changed dramatically. The rod and cocci bacteria have been replaced with bacteria structurally resembling Actinomyces type bacteria. Comparing Figure 2 to Figure 1 shows a dramatic change in the number of bacteria in response to direct medication treatment. Treatment usage was modified as pocket depths decreased.

$6 \mathrm{~mm}$ pockets or greater 4 times a day @ 15 minutes 3-6 $\mathrm{mm}$ pockets 3 times a day @15 minutes Less than $3 \mathrm{~mm}$ pockets 2 times a day @ 15 minutes Maintenance 1-2 times a day @ 15 minutes. The periodontal pockets were re-evaluated at regular intervals

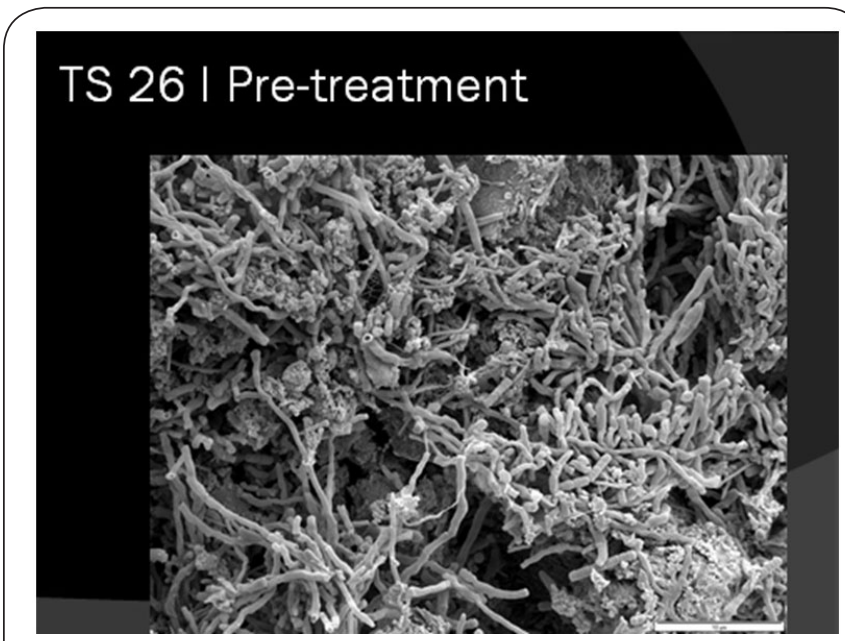

Figure 3. Shows the biofilm observed on the lingual periodontal pocket of tooth \#26. This SEM shows a luxurious amount of various bacteria involved in the biofilm.

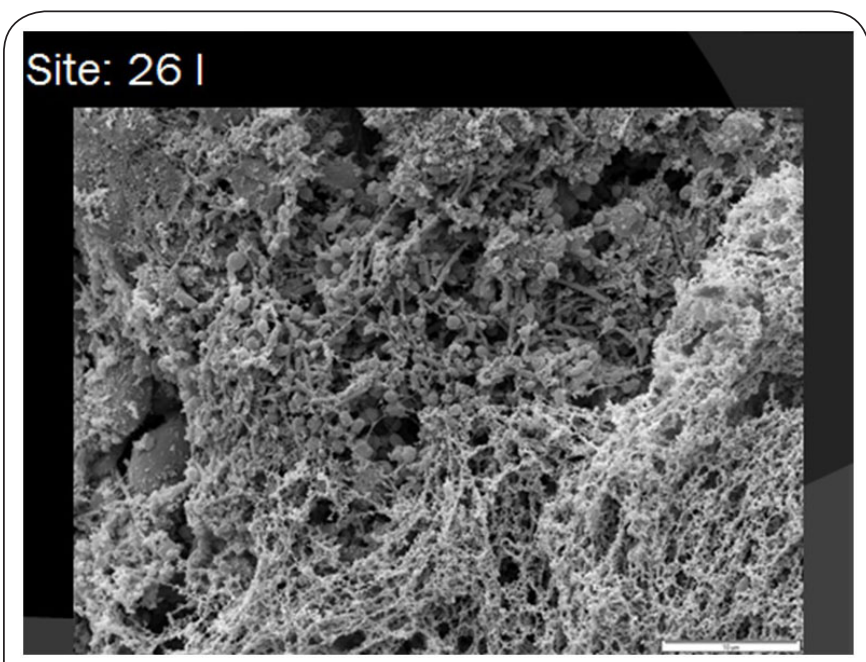

Figure 4. Shows the biofilm modified after two weeks of direct medication where $1.7 \%$ hydrogen peroxide gel was delivered to the periodontal pocket with the Perio Protect Method. Many of the bacteria can be seen with an irregular surface as the bacteria are being lysed through an oxidative/reduction modification.

and patient's treatments were modified as healing occurred. Patients with greater than $6 \mathrm{~mm}$ pockets reduced their usage from four time/day to three times a day when their deepest pocket was less than $6 \mathrm{~mm}$ deep and twice a day when the pockets measured less than $3 \mathrm{~mm}$. The deepest pocket determined the treatment frequency.

Research evaluating the efficacy of direct medication delivery of hydrogen peroxide was compared to direct medication delivery of hydrogen peroxide and Vibramycin as compared to scaling and root planing. These results showed hydrogen peroxide and Vibramycin were slightly more effective than hydrogen peroxide alone, which were both statistically sig- 
Keller et al. Oral Biology and Dentistry 2017,

\begin{tabular}{|llllllll|}
\hline Composite & G- Anaer & G+ Anaer & G- F An & G+ F An & G- Aero & G+ Aero & G- Micro \\
Before & 742 & 43 & 14 & 0 & 0 & 4 & 16 \\
During & 518 & 172 & 0 & 0 & 20 & 9 & 16 \\
After & 56 & 237 & 65 & 50 & 20 & 33 & 10 \\
\hline
\end{tabular}

Figure 5. Shows the composite numbers of all bacterial species determined by DNA analysis that are present following conventional mechanical treatment and homecare which are evident before treatment. These numbers can be compared to during and after direct medication treatment. The number of Gram negative obligate anaerobes was found to decrease as the number of Gram positive anaerobes and all facultative and aerobic bacteria increased during treatment. The total numbers of bacterial species decreased during the treatment and maintenance phase when compared to the before treatment numbers.

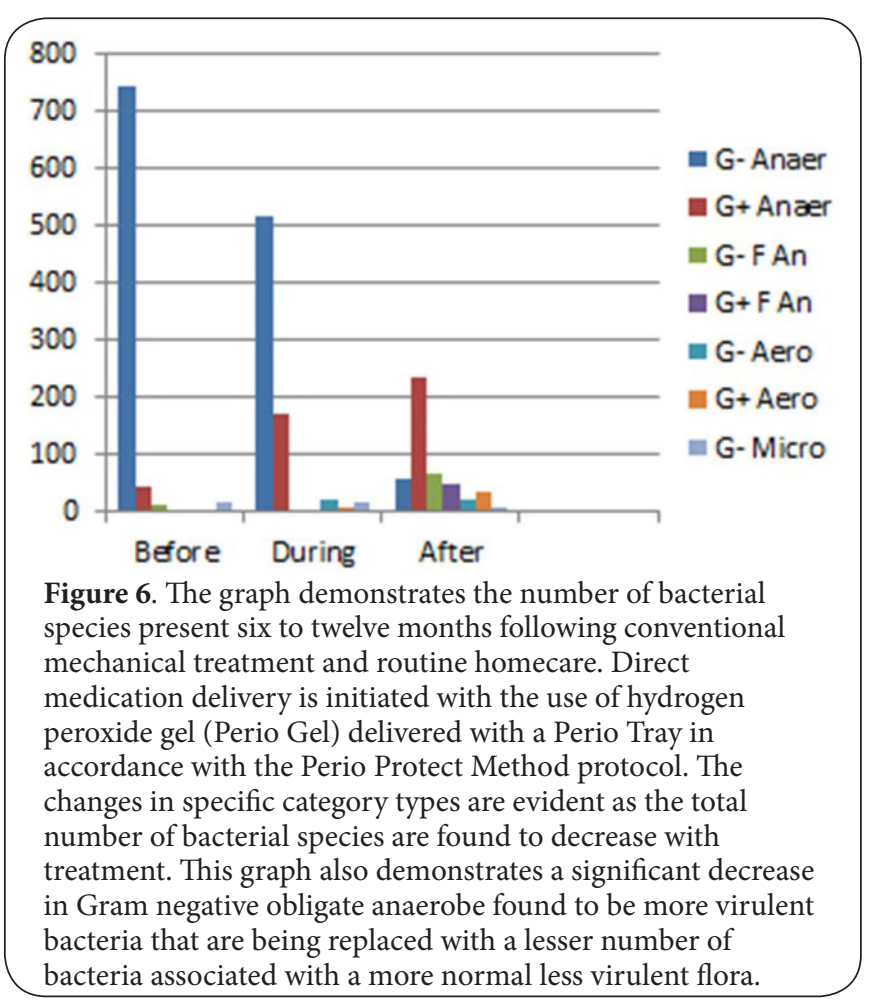

nificantly better than scaling and root planing $[29,30]$.

Samples for DNA analysis were taken from the same site at two weeks, one month and at three months. The computed DNA analysis of the samples enabled a comparison of bacteria taken prior to treatment to bacteria present during treatment to ascertain changes within the biofilm. Comparisons were evaluated as a change in the percentage of the bacteria and the appearance or disappearance of bacteria. The SEM analysis was used to determine the number of bacteria/unit area. The DNA analysis demonstrated the specific bacteria present in the biofilm. The prior to treatment population was compared to during and end of active treatment samples at the same site.

The direct medication delivery has been shown to manage the biofilm and resulted in a decrease of patient's periodontal conditions [33,34], and to decrease subgingival bacterial loads in periodontal pockets as deep as $8 \mathrm{~mm}$ [35]. After using the direct medication delivery for six weeks, scaling and root debridement therapy were provided. This involved both a cavitron and hand scaling and polishing. The patients then continued direct medication delivery via the customized tray and a final DNA analysis sample was taken at the end of prescribed treatment. The results of the DNA analysis are shown in Figures 5 and $\mathbf{6}$ where the number of bacteria and the bacterial species present are changed by the direct medication delivery. The predominant species before treatment are not the predominant species after treatment.

\section{Results}

The bacteria found in the patient's periodontal pockets in both the DNA analysis and SEM analysis had grown six to twelve months after conventional treatments and homecare. Figure 1, 3 and 5 demonstrate the type and numbers of bacteria that remained and regrew in the patient's periodontal pockets 6 to 12 months after conventional mechanical therapy and homecare.

Utilization of direct medication delivery demonstrated a significant change in both the type and number of bacteria present after treatment. Figure 2, 4, 5 and $\mathbf{6}$ demonstrate the type and number of bacteria after direct medication treatment. Comparing the results of conventional mechanical treatment and home care to direct medication delivery helps illustrate the differences between the treatments.

Figures 1 and $\mathbf{3}$ of the SEM analysis demonstrated the bacteria that were present in patients periodontal pocket 6 to 12 months after conventional mechanical treatment and conventional home care. Figures $\mathbf{2}$ and $\mathbf{4}$ show the bacteria that are left in the periodontal pocket as a result of direct medication delivery.

These figures show the bacteria in periodontal pockets changed dramatically during direct medication delivery treatment. The bacterial count/unit area with the SEM analysis demonstrated the greatest reduction in the number of bacteria within the first two weeks of treatment. The number of bacteria continued to decrease during treatment and remained decreased at the three month re-evaluation. 
Keller et al. Oral Biology and Dentistry 2017,

http://www.hoajonline.com/journals/pdf/2053-5775-5-1.pdf

doi: 10.7243/2053-5775-5-1

The DNA composition of the biofilm was different when comparing the type and the percentage of bacteria present six to twelve months after conventional mechanical treatment and home care to during and after direct medication delivery (Figures 5 and 6). In the DNA analysis, the number of bacteria found in the periodontal site following conventional care and before direct medication treatment was always greater than the number of bacteria found during direct medication treatment, which was greater than the number of bacteria found after treatment (Figure 6). The number of bacteria decreased in every site with the direct medication delivery by a negative $\log ^{2-4}$.

Following conventional treatment and homecare the predominant species in all periodontal pockets greater than $3 \mathrm{~mm}$ (Figures 5 and 6) were Gram negative obligate anaerobes with a lesser number of Gram positive anaerobes, Gram positive and negative facultative anaerobes and a few aerobic bacteria. The DNA analysis showed the composition of the bacteria changed during and after treatment. Figure 6 shows the change in the number of Gram negative obligate anaerobes which diminished as these were replaced with more Gram positive anaerobes, Gram positive and negative facultative anaerobes and aerobic bacteria during treatment. Figure 5 shows that by the end of treatment, the percent number of anaerobes has decreased as the percentage of facultative anaerobes and aerobic bacteria have increased. Figures 5 and $\mathbf{6}$ demonstrate the decreased numbers of bacterial species resulting from direct medication treatment.

\section{Discussion}

The results of this study are important because they show that direct medication deliveries of hydrogen peroxide, oxygen and Vibramycin into the periodontal pocket with a Perio Tray are effective in controlling the biofilm responsible for periodontal disease. The findings through SEM and DNA analyses provide optimal biofilm information.

Research shows that bacteria after conventional care can regrow to pre-treatment levels in a matter of days. SEM analysis shows the number of bacteria after direct medication delivery is reduced by a - $\log ^{2-4}$, and remains at this decreased number for up to three months. Decreasing the number of pathogens should decrease the host inflammatory response.

DNA analysis determined a change in the predominant species and types of bacteria before and after treatment. The predominant more virulent pre-treatment species; Gram negative oblige anaerobes, are replaced by fewer less virulent Gram positive anaerobes, Gram positive and negative facultative anaerobes and aerobic bacteria. Fewer less virulent bacteria would equate with a decreased inflammatory response which should be beneficial for an improved patient's health.

\section{Conclusions}

The size of this clinical study is small, which affects statistical significance. However, the findings of this study have tremendous impact on the clinical significance for present and future periodontal treatments when combined with conventional care. Direct medication delivery provides better biofilm control than conventional care.

Direct medication delivery of hydrogen peroxide, oxygen and Vibramycin delivered subgingival and interproximal reduces the number of bacteria by a negative $\log ^{2-4}$. The more virulent bacteria that were the predominant species in the biofilm prior to direct medication delivery are changed and are replaced by a less virulent type of bacteria after direct medication delivery. Treatments that result in fewer bacteria that are less virulent and comprise a more normal bacterial flora should be beneficial for the patient.

\section{Competing interests}

The authors declare that they have no competing interests.

\section{Authors' contributions}

\begin{tabular}{|l|c|c|}
\hline Authors' contributions & DCK & MB \\
\hline Research concept and design & $\checkmark$ & $\checkmark$ \\
\hline Collection and/or assembly of data & $\checkmark$ & $\checkmark$ \\
\hline Data analysis and interpretation & $\checkmark$ & $\checkmark$ \\
\hline Writing the article & $\checkmark$ & $\checkmark$ \\
\hline Critical revision of the article & $\checkmark$ & $\checkmark$ \\
\hline Final approval of article & $\checkmark$ & $\checkmark$ \\
\hline Statistical analysis & $\checkmark$ & $\checkmark$ \\
\hline
\end{tabular}

\section{Acknowledgement}

Perio Protect provided Perio Trays and Perio Gel for these evaluations.

\section{Publication history}

Editor: Cássio do Nascimento, University of Sao Paulo, Brazil. Received: 05-Nov-2016 Final Revised: 16-Jan-2017

Accepted: 06-Feb-2017 Published: 14-Feb-2017

\section{References}

1. Roberts SK, Bass C, Brading M, Lappin-Scott H and Stoodley P. Biolm information and structure: what's new? In: New-man HN, Wilson M, editors. Dental plaque revisited. Cardiff: Bioline. 1999; 15-35.

2. Kang CH, Nam YD, Chung WH, Quan ZX, Park YH, Park SJ, Desmone R, Wan XF and Rhee SK. Relationship between genome similarity and DNA-DNA hybridization among closely related bacteria. J Microbiol Biotechnol. 2007; 17:945-51. | Article | PubMed

3. Center for Biofilm Engineering. | Website

4. McCann KS. The diversity-stability debate. Nature. 2000; 405:228-33. | Article | PubMed

5. Paster BJ, Olsen I, Aas JA and Dewhirst FE. The breadth of bacterial diversity in the human periodontal pocket and other oral sites. Periodontol 2000. 2006; 42:80-7. | Article | PubMed

6. Huang R, Li M and Gregory RL. Bacterial interactions in dental biofilm. Virulence. 2011; 2:435-44. | Article | PubMed Abstract | PubMed FullText

7. Saroch M Periobasics.com. | Website

8. Trost B, Pajon R, Jayaprakash $T$ and Kusalik A. Comparing the similarity of different groups of bacteria to the human proteome. PLoS One. 2012; 7:e34007. I Article | PubMed Abstract | PubMed FullText

9. Duerden BI. Virulence factors in anaerobes. Clin Infect Dis. 1994; 18 Suppl 4:S253-9. | PubMed 
Keller et al. Oral Biology and Dentistry 2017,

10. Anaerobic bacteria. Infectious Disease and Antimicrobial Agents. | Article

11. Jousimies-Somer $\mathrm{H}$, Summanen $\mathrm{P}$ and Citron DM et al. Anaerobic bacteriology manual 6th ed. Belmont, CA: Star Publishing Co. 2002.

12. Brook I. Chapter 9 Abscesses. Polymicrobial diseases. I Article

13. Uzel NG, Teles FR, Teles RP, Song XQ, Torresyap G, Socransky SS and Haffajee AD. Microbial shifts during dental biofilm re-development in the absence of oral hygiene in periodontal health and disease. J Clin Periodontol. 2011; 38:612-20. | Article | PubMed Abstract | PubMed FullText

14. Colombo AP, Teles RP, Torres MC, Rosalem W, Mendes MC, Souto RM and Uzeda M. Effects of non-surgical mechanical therapy on the subgingival microbiota of Brazilians with untreated chronic periodontitis: 9-month results. J Periodontol. 2005; 76:778-84. I Article I PubMed

15. Palmer RJ and Caldwell DE. Effects of mechanical dental therapy. J Micro methods. 1995; 24:171-82.

16. Teles FR, Teles RP, Uzel NG, Song XQ, Torresyap G, Socransky SS and Haffajee AD. Early microbial succession in redeveloping dental biofilms in periodontal health and disease. J Periodontal Res. 2012; 47:95-104. I Article | PubMed Abstract | PubMed FullText

17. Ibid.

18. CDC. The Use and Handling of Toothbrushes. | Website

19. Costa MR, da Silva VC, Miqui MN, Colombo AP and Cirelli JA. Effects of ultrasonic, electric, and manual toothbrushes on subgingival plaque composition in orthodontically banded molars. Am J Orthod Dentofacial Orthop. 2010; 137:229-35. | Article | PubMed

20. Petersilka GJ, Ehmke B and Flemmig TF. Antimicrobial effects of mechanical debridement. Periodontol 2000. 2002; 28:56-71. | Article | PubMed

21. Bradshaw DJ, Marsh PD, Watson GK and Allison C. Role of Fusobacterium nucleatum and coaggregation in anaerobe survival in planktonic and biofilm oral microbial communities during aeration. Infect Immun. 1998; 66:4729-32. | Article | PubMed Abstract | PubMed FullText

22. Acharya T. Oxygen requirement for pathogenic bacteria. I Website

23. How KY, Song KP and Chan KG. Porphyromonas gingivalis: An Overview of Periodontopathic Pathogen below the Gum Line. Front Microbiol. 2016; 7:53. | Article | PubMed Abstract | PubMed FullText

24. Schaudinn C, Gorur A, Keller D, Sedghizadeh PP and Costerton JW. Periodontitis: an archetypical biofilm disease. J Am Dent Assoc. 2009; 140:978-86. | Article | PubMed

25. Schaudinn C et al. Manipulation of the microbial ecology of the periodontal pocket. World Dental. 2010; 2:14-8

26. Ghotaslou R and Salahi B. Effects of oxygen on in-vitro biofilm formation and antimicrobial resistance of Pseudomonas aeruginosae. Pharm Sci. 2013; 19:96-9. I Pdf

27. Acharya T. Oxygen requirements for pathogenic bacteria. Microbe online. I Website

28. Nyvad B and Kilian M. Microbiology of the early colonization of human enamel and root surfaces in vivo. Scand J Dent Res. 1987; 95:369-80. | PubMed

29. Chandki R, Banthia P and Banthia R. Biofilms: A microbial home. J Indian Soc Periodontol. 2011; 15:111-4. | Article | PubMed Abstract | PubMed FullText

30. Teles FR, Teles RP, Sachdeo A, Uzel NG, Song XQ, Torresyap G, Singh M, Papas A, Haffajee AD and Socransky SS. Comparison of microbial changes in early redeveloping biofilms on natural teeth and dentures. J Periodontol. 2012; 83:1139-48. | Article | PubMed Abstract | PubMed FullText

31. Dunlap T, Keller DC, Marshall MV, Costerton JW, Schaudinn C, Sindelar B and Cotton JR. Subgingival delivery of oral debriding agents: a proof of concept. J Clin Dent. 2011; 22:149-58. I PubMed

32. Putt MS and Proskin HM. Custom tray application of peroxide gel as an adjunct to scaling and root planing in the treatment of periodontitis: a randomized, controlled three-month clinical trial. J Clin Dent. 2012;
23:48-56. | PubMed

33. Putt MS, Mallatt ME, Messmann LL and Proskin HM. A 6-month clinical investigation of custom tray application of peroxide gel with or without doxycycline as adjuncts to scaling and root planing for treatment of periodontitis. Am J Dent. 2014; 27:273-84. | PubMed

Citation:

Keller DC and Buechel M. Direct medication delivery modifies the periodontal biofilm. Oral Biol Dent. 2017; 5:1. http://dx.doi.org/10.7243/2053-5775-5-1 